\title{
On the effect of image denoising on galaxy shape measurements
}

\author{
G. Nurbaeva, F. Courbin, M. Gentile, and G. Meylan
}

\author{
Laboratoire d'astrophysique, École Polytechnique Fédérale de Lausanne (EPFL), Observatoire de Sauverny, 1290 Versoix, \\ Switzerland
}

Received 21 January 2011 / Accepted 24 April 2011

\begin{abstract}
Weak gravitational lensing is a very sensitive way of measuring cosmological parameters, including dark energy, and of testing current theories of gravitation. In practice, this requires exquisite measurement of the shapes of billions of galaxies over large areas of the sky, as may be obtained with the EUCLID and WFIRST satellites. For a given survey depth, applying image denoising to the data both improves the accuracy of the shape measurements and increases the number density of galaxies with a measurable shape. We perform simple tests of three different denoising techniques, using synthetic data. We propose a new and simple denoising method, based on wavelet decomposition of the data and a Wiener filtering of the resulting wavelet coefficients. When applied to the GREAT08 challenge dataset, this technique allows us to improve the quality factor of the measurement ( $Q$; GREAT08 definition), by up to a factor of two. We demonstrate that the typical pixel size of the EUCLID optical channel will allow us to use image denoising.
\end{abstract}

Key words. methods: data analysis - techniques: image processing - gravitational lensing: weak - dark energy cosmological parameters - methods: statistical

\section{Introduction}

The observed accelerated expansion of the Universe (Riess et al. 1998; Perlmutter et al. 1999) can currently be explained by either the existence of a repulsive force associated with so-called "dark energy", or an erroneous description of gravity by General Relativity on large spatial scales (for a review, see Frieman et al. 2008). Both explanations have profound implications for our understanding of cosmology and physics in general and are the main motivations of future large cosmological surveys. These surveys, such as the ESA EUCLID satellite project (Réfrégier et al. 2010) combine several complementary cosmological probes to constrain the cosmological parameters, including the dark energy equation of state parameter $w(z)$ and its evolution with redshift.

The main cosmological probe to be employed by EUCLID is weak gravitational lensing, also known as cosmic shear. The observational signature of cosmic shear is an apparent distortion of the image of distant galaxies under the influence of gravitational lensing by a foreground potential well. The exact way in which the galaxies are distorted is very sensitive to the dark matter and dark energy distributions in the foreground large-scale structures, hence providing an efficient tool for cosmological measurements. While the first detections of cosmic shear are already a decade old and were performed on data with relatively limited field of view and depth (Bacon et al. 2000; van Waebeke et al. 2000; Wittman et al. 2000), the use of cosmic shear in terms of cosmological applications requires a major space survey. However, the effectiveness of the method in constraining cosmology relies on image processing techniques that measure the shapes of individual galaxies in the most accurate possible way. These techniques must provide solutions to the four following problems: (i) the degradations caused by the dominating Poisson noise; (ii) the sampling adopted to represent the data; (iii) the convolution by the instrumental point spread function (PSF) and its possible variations across the field of view; and (iv) the measurement of the cosmic shear itself and its power spectrum from all the galaxy shape measurements, i.e., billions of galaxies.

The techniques currently in use to measure cosmic shear are sufficient to detect it and even sometimes to reconstruct the 3D mass map of large-scale structures (e.g., Massey et al. 2007a) but it is estimated that a tenfold improvement in the precision of galaxy shape measurements is needed to place stringent constraints on cosmological models. Thanks to both the STEP programs (Heymans et al. 2006; Massey et al. 2007b) and the GREAT08 ${ }^{1}$ challenge (Bridle et al. 2010), the lensing community has made excellent progress toward meeting this goal. However, even the most successful shear measurement methods see their accuracy decrease significantly under high noise conditions. It is therefore of interest to develop suitable denoising techniques that are capable of solving the difficult problem of removing noise without compromising the fragile shear signal.

In the present work, we focus on the effects of denoising and pixelisation on shape measurement. Both are closely connected since the spatial frequencies contained in the galaxy images change with the adopted pixel size, but not the noise frequency. Moreover, for a given exposure time, changing the pixel size affects the signal-to-noise ratio $(S N R)$ of the data.

For this reason, it is important to explore the large parameter space of the problem and to weight the relative impacts of different samplings and $S N R$ s on the shear measurement. To investigate this problem, we use sets of synthetic galaxies with known ellipticities, for different resolutions and samplings spanning a range of observational setups. Using these data, as well as a subset of the GREAT08 data (Bridle et al. 2010), we test the performance of three popular denoising algorithms: median filtering, Wiener filtering, and discrete wavelet transform (DWT). We also propose a new denoising method based on a combination of wavelet transform and Wiener filtering.

${ }^{1}$ http://www.greatchallenges.info/ 


\section{Methods}

\subsection{Median filter}

The median filter is a nonlinear denoising technique widely used in digital image processing. Apart from its simplicity, median filtering has two important properties: firstly, it is particularly effective for images corrupted by Poisson noise and secondly, it preserves edges in images.

A median filter works by sliding a box of given size $(3 \times 3$ pixels in our case $)$ over the image, replacing the central value by the median of its neighboring pixels (Arce 2005; Arias-Castro \& Donoho 2009).

\subsection{Wiener filter}

The Wiener filter uses a least mean squares filtering algorithm (Wiener 1949) based on a stochastic framework that minimizes the mean square error in the noise. The Wiener filter has become a classical signal smoothing technique and is widely used in signal processing (Khireddine et al. 2007; Press et al. 2007). In our study, we use the following simplified algorithm.

If we define $I$ to be the input brightness of the pixel of the noisy image, the output brightness $I_{\text {wiener }}$ of the denoised pixel is then given by

$I_{\text {wiener }}=\left\{\begin{aligned} I\left(1-\sigma_{n}^{2} / \sigma_{w}^{2}\right), & \sigma_{w}^{2} \geq \sigma_{n}, \\ 0, & \sigma_{w}^{2}<\sigma_{n},\end{aligned}\right.$

where $\sigma_{n}$ is the estimated mean standard deviation of the noise in the image and $\sigma_{w}^{2}$ is an average variance in the pixel values calculated in a local window of $3 \times 3$ pixels.

The algorithm implemented in this work uses the scientific libraries (SciPy community 2010) available with the Python programming language.

\subsection{Discret wavelet transform (DWT)}

Wavelet analysis is an efficient and fast computational technique widely used for data compression and noise reduction (Bruce et al. 1996). In our study, we apply the 2D discrete wavelet transform (DWT). Denoising in wavelet space involves three steps: (i) linear forward wavelet decomposition, (ii) shrinkage of wavelet coefficients, and (iii) linear inverse wavelet reconstruction.

As the basis function we adopt the Haar wavelet, which is orthogonal and computationally simple. The latter property is of primary importance to preserving shape invariance.

The Haar wavelet is defined by two basic functions: a scaling function $\phi$ and a wavelet function, called the mother wavelet $\psi$. The set of basic functions for the 1D case is given by

$$
\left\{\begin{array}{l}
\phi_{i}^{j}(x)=\phi\left(2^{k-j} x-i\right) \\
\psi_{i}^{j}(x)=\psi\left(2^{k-j} x-i\right), \quad i=0,1,2, \ldots, N-1
\end{array}\right.
$$

where $N=2^{k}-j$ is the number of wavelet coefficients, which also defines the size of the subband of a given decomposition level $j$, where $k$ is the coarsest level. The scaling function and the mother wavelet are defined as follows:

$\phi(x)=\left\{\begin{array}{l}1,0 \leq x<1 \\ 0, \text { otherwise }\end{array} \quad \psi(x)=\left\{\begin{array}{r}1,0 \leq x<1 / 2 \\ -1,1 / 2 \leq x<1 \\ 0, \quad \text { otherwise }\end{array}\right.\right.$

To decompose a two-dimensional image, the coefficients are obtained by multiplying the one-dimensional scaling and the wavelet functions both in the horizontal and vertical directions. For each resolution level, the image is devided into four images of coefficients, called subbands. The first, often labeled $L L$ (low-low), contains the main (low)-frequency features of the signal. The three others are dominated by the noise in the horizontal direction, $H L$ (high-low), vertical direction, $L H$ (low-high), and diagonal direction, $H H$ (high-high). Iterating the described scheme (Eq. (2)), one can obtain an image sequence with a cascading structure as illustrated in Fig. 2.

The most important part of the DWT denoising technique is the wavelet shrinkage, which drives the efficiency of denoising. The wavelet shrinkage is applied to the subbands associated with the noise: $H L, L H$, and $H H$. The classical way to suppress the noise by shrinking the wavelet coefficients is to apply a threshold to the wavelet coefficients. There are two types of thresholding algorithms: soft and hard thresholding. For a given value of the threshold $T$, hard thresholding sets all coefficients less than $T$ to zero. For the soft thresholding, $T$ is subtracted from all coefficients greater than $T$ (see e.g., Vetterli \& Kovacevic 1995).

\subsubsection{Bayes thresholding}

The wavelet shrinkage step depends heavily on the choice of the thresholding scheme. Popular thresholding methods include Stein's unbiased risk estimate (SURE; Donoho \& Johnstone 1994) and universal thresholding (Donoho \& Johnstone 1995), the latter depending on image size. A more efficient thresholding scheme is the Bayes wavelet threshold proposed by Chang (2000), which uses a different threshold level for each of the three $H L, L H$, and $H H$ noise subbands. This adaptive threshold, $T_{\text {Bayes }}$, is computed to be

$T_{\text {Bayes }}=\frac{\sigma_{n}^{2}}{\sigma_{x}}$,

where $\sigma_{x}$ is the standard deviation in the noiseless coefficients in a given subband, which can be estimated as

$\sigma_{x}=\sqrt{\max \left(\sigma_{y}^{2}-\sigma_{n}^{2}, 0\right)}$

where $\sigma_{y}^{2}$ is the variance in the coefficients in a subband. If $\sigma_{x}=0$ then $T_{\text {Bayes }}$ diverges to infinity, meaning that all coefficients in the corresponding subband must be set to zero.

\subsubsection{Combined DWT-Wiener filter: a new thresholding scheme}

The DWT-Bayes thresholding gives good results for generalpurpose imaging of relatively small dynamical range. However, it is quickly limited when dealing with astronomical images where the intensity levels vary sharply on spatial scales in the order of the PSF size. Standard thresholding, where $T$ is the same across each wavelet subband, degrades galaxy shapes with steep intensity profiles, especially when images are critically sampled. This is simply because the light profile between two neighboring pixels is much steeper than the estimated noise per pixel, $\sigma_{n}$. The resulting denoised image is therefore too smooth in rather flat areas such as the image background, and too noisy in areas of larger dynamical range, i.e., where galaxies lie. This has a significant effect on the shape measurement accuracy. 

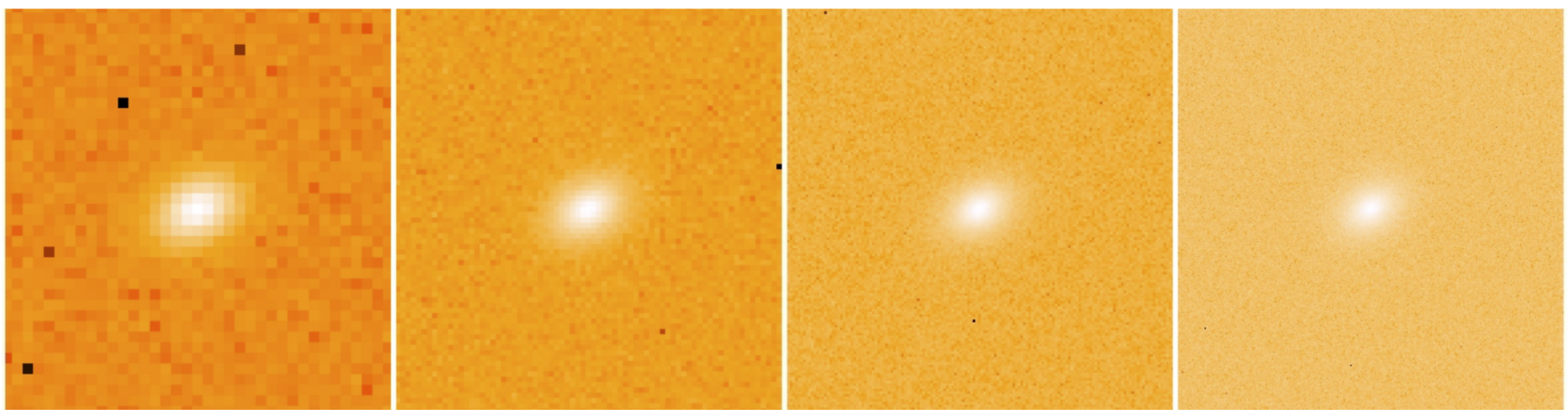

Fig. 1. Artificial galaxies of different resolutions. From left to right the stamp size is 40,80,160 and 320 pixels on a side, corresponding to a mean SNR per pixel of $1.5,0.75,0.37$ and 0.19 , respectively. The first two samplings on the left correspond to the "realistic sampling" and the "small sampling" cases, respectively (see text).

For this reason, we propose a simple and effective method that combines the Haar DWT and the classical Wiener algorithms in the following way:

1. we decompose the image and calculate the $H L_{j}, L H_{j}$, and $\mathrm{HH}_{j}$ subbands of wavelet coefficients for all resolution levels $j=1,2, \ldots, k$, where $k$ is the coarsest level;

2. we then modify the wavelet subbands $H L_{j}, L H_{j}$, and $H H_{j}$ using the kernel Wiener algorithm (Eq. (1));

3. we finally apply the inverse DWT to the modified wavelet coefficient to reconstruct the denoised image.

In other words, we apply the Wiener method to the wavelet coefficients rather than to the data pixels themselves. We show in the following that, for images of faint and small galaxies, this simple method not only conserves the shape of the galaxies, but also improves their measurement.

\section{Synthetic data}

We use a set of 10000 simulated galaxies in order to (i) test the effect of the different denoising techniques on the shape measurement and (ii) estimate how this behavior depends on the sampling and the SNR of the data. Each galaxy is represented by a Sersic profile of known ellipticity and Sersic index. This profile is sampled on five different grids of pixels. In doing this, we keep the size of the galaxy fixed on the plane of the sky.

Our finest sampling has galaxies with a full-width-halfmaximum of $F W H M=17.3$ pixels. Each of the 10000 galaxy images is represented on a stamp of $320 \times 320$ pixels. We then degrade the sampling by a factor of two, four times in a row, to produce galaxy images with $F W H M \sim 17,9,5,3$ pixels, which correspond, respectively, to image sizes of $320,160,80$, and 40 pixels on a side.

We then add Poisson noise to the simulated data, assuming that the data are sky-dominated, i.e, to a good approximation the amplitude of the noise is the same for all pixels across the galaxy image. We generate noisy images that mimic those of the GREAT08 challenge (Bridle et al. 2010) with a standard deviation set to the value of $\sigma_{n}=1000$ for all pixels. Before adding the noise, we scale the galaxy images so that we probe a range of realistic $S N R$. In the rest of the paper, we refer to the mean $S N R$ per pixel, i.e.,

$$
S N R=\sum_{j=1}^{N} \frac{I_{j}}{N \times \sigma_{n}},
$$

where $I_{j}$ is the image value at pixel $j$ and $N$ is the total number of pixels in the image. Since the exposure time is limited in real sky surveys, improving the sampling of the data is done to the cost of a lower $S N R$ per pixel. All our simulated images are computed for a fixed integration time.

We use four different samplings, characterized by the typical $F W H M$ of the simulated galaxies. The first two samplings have $F W H M \sim 3$ and 5 pixels which are typical values for a space mission such as EUCLID. In the following, we refer to these as "real sampling" data. We also use two smaller samplings of $F W H M \sim 9$ and 17 pixels. We refer to these as "small sampling" data. Figure 1 give examples of simulated images for the same realization of a galaxy. Our simulations span the $S N R$ range between 0.05 to 4.0 .

For reference, the SNR of the GREAT08 challenge data range from 0.68 to 2.6 in the "low noise" dataset and from 0.003 to 0.38 for the high noise dataset. The $F W H M$ of the galaxies in GREAT08 varies from 1.1 to 14.5 pixels.

\section{Results and discussion}

\subsection{Denoising efficiency for synthetic galaxies}

Using the set of 10000 artificial galaxies described in Sect. 3, we test the performance of the four methods under different SNR and sampling conditions. Examples of denoised images are shown in Fig. 3, where it is immediately seen that the four methods behave very differently.

The median filtering has a kernel size of $3 \times 3$ pixels. As a consequence, when the sampling changes, the spatial frequencies removed by the filter change with respect to the ones contained in the galaxy. When the sampling becomes coarse enough, the frequencies removed by the filter are close to those contained in the galaxy. This is seen in Fig. 3, where the size of the granulation in the noise becomes larger as the pixel size becomes larger (right column of the figure). For this reason, the performance of the median filtering are expected to degrade quickly in cases of coarse sampling.

The Wiener filter is a low-pass filter, which translates in Fig. 3 into a strong "flattening" of the sky noise but almost no denoising of the galaxy itself. The wavelet method is fully local and adaptive, explaining the patchy aspect of the galaxy in Fig. 3: low frequency signals are represented using a limited number of coefficients and a high frequency requires more coefficients

Finally, we show the results of both DWT-Wiener and DWT-Bayes methods. As explained earlier, the standard DWTBayes thresholding is not effective in removing noise from high 

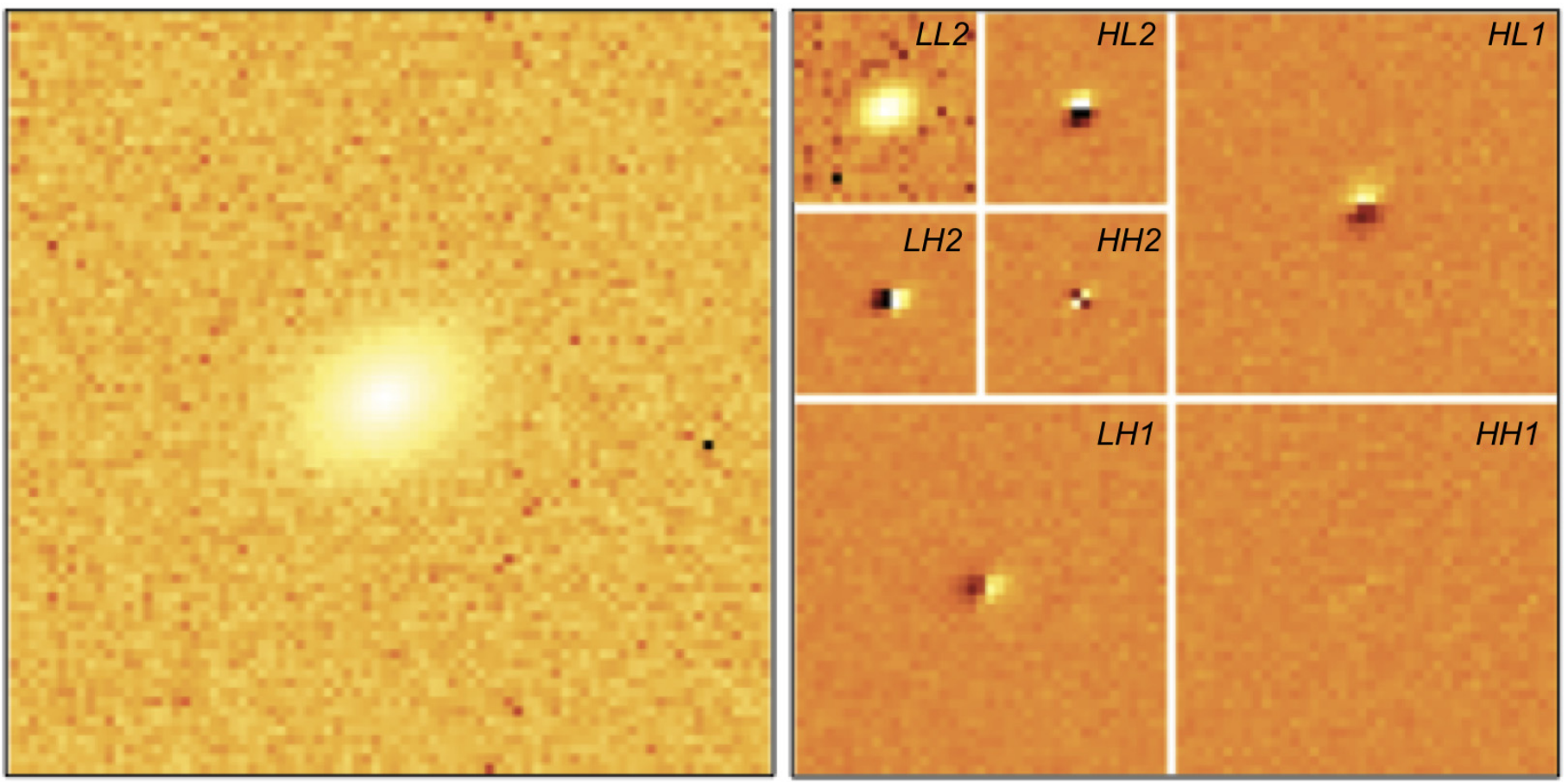

Fig. 2. DWT decomposition of the galaxy image, with Poisson noise. Left: original galaxy image. Right: two consecutive resolution levels, as explained in Sect. 2.3.

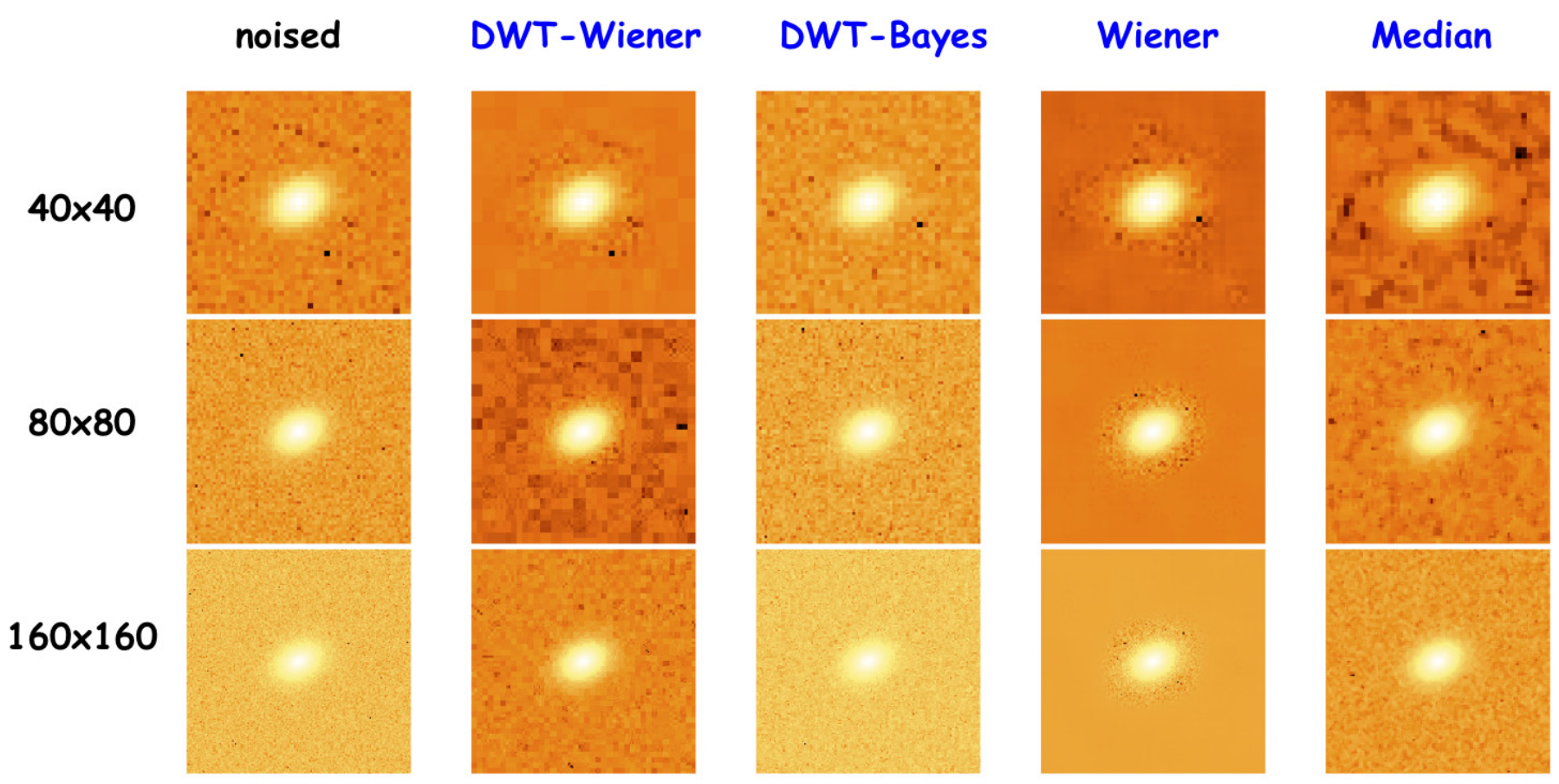

Fig. 3. Examples of different denoising methods, using simulated data with three different samplings.

dynamical range data such as astronomical images. In the particular case of galaxy images, using a fixed threshold for all the wavelet subbands tends to degrade the galaxy shape in areas where the difference in brightness between neighboring pixels is much higher than the standard deviation of the Poisson noise. In other words, the threshold is too high for the image background, leading to an excessive smoothing, whereas the same threshold proves too small for the high intensity pixels of the galaxy image.
This phenomenon is clearly visible in the image denoised with the DWT-Bayes threshold (middle column of Fig. 3), where the results of the denoising process leaves the original image almost unchanged. This is unfortunate because preserving the original intensity profile of the galaxy is essential for the accuracy of the measurement of its shape. Removing noise effectively while preserving the galaxy shape requires a very delicate denoising approach. 


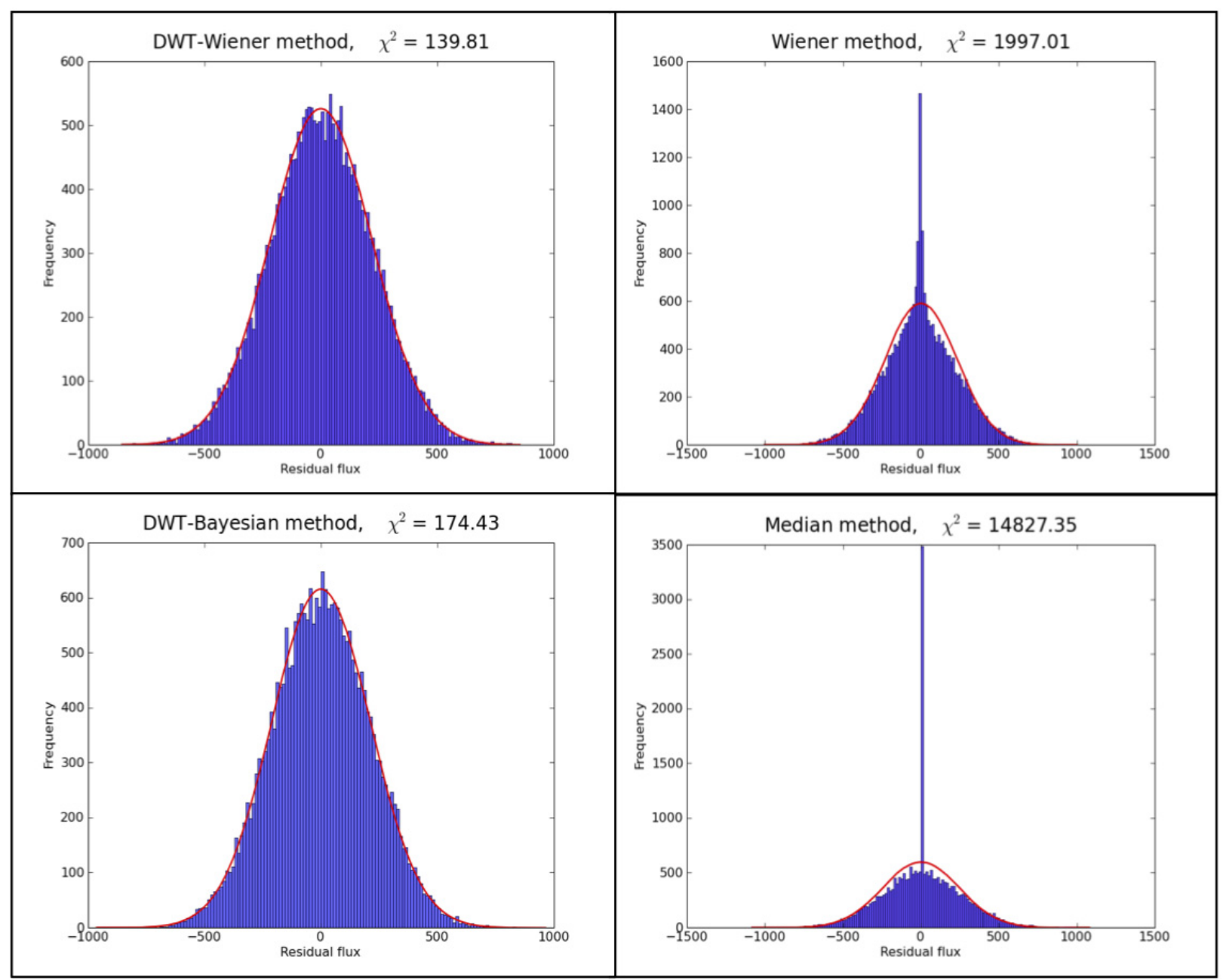

Fig. 4. Effect of the four denoising methods on the noise properties of the original data. Each panel shows the normalized histograms of residual images, i.e., the difference between the original noisy data and the denoised data. The red line shows the best-fit Gaussian. In each case, the $\chi^{2}$ of the fit is indicated. The mean $S N R$ in the image selected to compute the histogram is $S N R=0.38$.

The DWT-Wiener method proposed in this paper removes noise according to the local gradient of luminosity, resulting in a more adaptive and local denoising process than DWT-Bayes. The advantage of DWT-Wiener in comparison to the classical Wiener filter is that DWT-Wiener is applied to high-frequency subbands coefficients only, which are those associated with noise, thus preserving the low frequency subband that contains the main features of the signal.

The effect of the four methods on the noise properties of the original data is seen more quantitatively in Fig. 4. In this figure, we choose a galaxy realization with a $S N R=0.38$ and sampling of $F W H M=5$ pixels (realistic sampling). We then compute the difference between the original noisy image and the denoised image, as obtained from the four methods and plot histograms of these distributions in Fig. 4. A method that does not affect the noise properties of the original data should ideally yield a perfectly Gaussian histogram. Only the two local denoising methods using wavelets possess this important property.

For each denoising method, we thus compute the $\chi^{2}$ statistics of its normalized histogram as (Press et al. 2007)

$\chi^{2}=\sum_{i} \frac{\left(N_{i}-n_{i}\right)^{2}}{n_{i}}$,

where $N_{i}$ is the number of pixels observed in the $i$ th bin, and $n_{i}$ is the number for an expected Gaussian distribution.
Our new DWT-Wiener method has the smallest $\chi^{2}$, which indicates that its residuals represent the best fit to a normal distribution. We now test the performance of the four methods on the sets of 10000 simulated galaxies described in Sect. 3, without considering the effect of convolution by the PSF. This is done by directly comparing the ellipticity measured for the galaxies before and after denoising. Using 10000 galaxies ensures that an ellipticity measurement is accurate to $1 \%$, which is sufficient for our purposes. In this work, the galaxy ellipticity $e=e_{1}+\mathrm{i} e_{2}$ was estimated as

$e=\frac{Q_{11}-Q_{22}+2 \mathrm{i} Q_{12}}{Q_{11}+Q_{22}}$,

where $\quad Q_{m n}, \quad(m, n \in\{1,2\}) \quad$ are the second-order quadrupole moments of the galaxy surface brightness (Bartelmann \& Schneider 2001).

We define the accuracy of each of the denoising methods as the inverse of the root mean square deviation (RMSD)

$R M S D=\sqrt{\frac{1}{N} \sum_{1}^{N}\left(e_{i}-e_{i}^{*}\right)^{2}}$,

where $e_{i}$ and $e_{i}^{*}$ denote estimated and true galaxy ellipticities, respectively. 

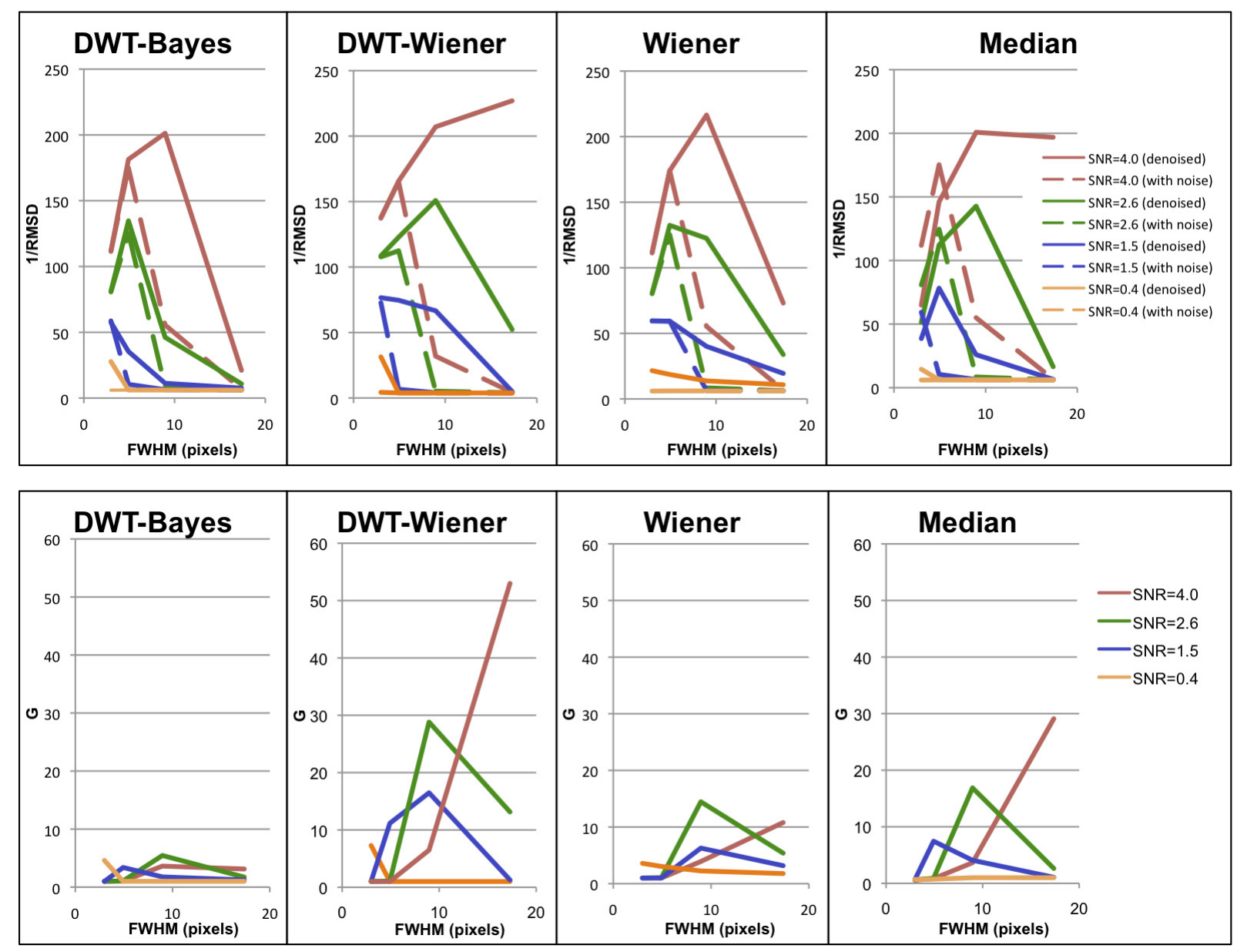

Fig. 5. Top panels: accuracy, 1/RMSD, of the shape measurement using the four denoising methods (solid curves). The dashed curves show for comparison the results obtained with the data before denoising. In each panel, the color code indicates different noise levels. Bottom panels: gain ratio, $G$, for the four denoising methods. The curves are the ratio of the solid to dashed curves shown in the top panels.

To evaluate the effectiveness of the denoising methods in providing improved measurement over the original data, we also define a gain ratio, $G$, as the ratio of the shape measurement error using the noisy data, to the same quantity using the denoised data

$G=\frac{R M S D_{\text {original }}}{R M S D_{\text {denoised }}}$

The results are summarized in Table 1 and Fig. 5. With no big surprise, all methods lead to high accuracy measurements as soon as excellent sampling and high SNR are available. However, when the $S N R$ decreases, the performance of all methods drops sharply. This means that there is no reason to improve the sampling indefinitely without compensating for an increased $S N R$, i.e., exposure time. This also means that the interest in denoising exceeds the improvement for poor $S N R$ data. Performances improve with high $S N R$ data (red curves in Fig. 5, hence showing that denoising is as important for well exposed galaxies as for galaxies barely measurable in the original data.

In coarse sampling conditions, the median filter degrades shape measurement instead of improving it. This is illustrated in Fig. 5 by the dashed curves systematically being above the solid ones. In Table 1 , the gain ratio factor, $\xi$, is indeed lower than 1 . This is because the frequencies removed by the median filter cannot be easily controlled and depend on the size of the objects with respect to the size of the median kernel. This makes the
Table 1. Gain ratio $(G$; see text) for the four denoising methods under different $S N R$ and sampling conditions.

\begin{tabular}{rrrrrrr}
\hline \hline$S N R$ & $\begin{array}{r}\text { Stamp } \\
\text { size }\end{array}$ & $\begin{array}{r}\text { FWHM } \\
\text { galaxies }\end{array}$ & Median & Wiener & $\begin{array}{r}\text { DWT } \\
\text { Bayes }\end{array}$ & $\begin{array}{r}\text { DWT } \\
\text { Wiener }\end{array}$ \\
\hline 4.00 & 40 & 2.99 & 0.58 & 1.00 & 1.00 & 1.01 \\
2.00 & 80 & 4.92 & 0.83 & 1.00 & 1.04 & 1.01 \\
1.00 & 160 & 8.96 & 3.65 & 3.91 & 3.62 & 6.49 \\
0.50 & 320 & 17.30 & 29.10 & 10.80 & 3.12 & 52.98 \\
\hline 2.60 & 40 & 2.99 & 0.64 & 1.00 & 1.01 & 1.01 \\
1.30 & 80 & 4.92 & 0.90 & 1.06 & 1.08 & 1.10 \\
0.65 & 160 & 8.96 & 16.87 & 14.47 & 5.45 & 28.81 \\
0.33 & 320 & 17.30 & 2.63 & 5.40 & 1.73 & 13.15 \\
\hline 1.50 & 40 & 2.99 & 0.65 & 1.00 & 0.98 & 1.05 \\
0.75 & 80 & 4.92 & 7.45 & 1.00 & 3.38 & 11.17 \\
0.38 & 160 & 8.96 & 4.08 & 6.28 & 1.77 & 16.48 \\
0.19 & 320 & 17.30 & 1.07 & 3.18 & 1.24 & 1.28 \\
\hline 0.40 & 40 & 2.99 & 0.69 & 3.59 & 2.67 & 7.28 \\
0.20 & 80 & 4.92 & 0.74 & 3.05 & 0.31 & 1.01 \\
0.10 & 160 & 8.96 & 1.00 & 2.25 & 0.99 & 1.00 \\
0.05 & 320 & 17.30 & 1.00 & 1.79 & 1.00 & 1.00 \\
\hline
\end{tabular}

Notes. The $S N R$ is per pixel (Eq. (6)).

use of the median filter very hazardous in general, in spite of its good performance in small sampling conditions. A similar trend is found for the Wiener filter in realistic sampling conditions, 
Table 2. $Q$ factor for KSB deconvolution for 15 galaxy sets of GREAT08 low noise-blind (LNBL) and 100 sets of GREAT08 real noise-blind (RNBL).

\begin{tabular}{lcccc}
\hline \hline Dataset & $\alpha$ & Original & $\begin{array}{c}\text { DWT-Bayes } \\
\text { denoising }\end{array}$ & $\begin{array}{c}\text { DWT-Wiener } \\
\text { denoising }\end{array}$ \\
\hline & 0.01 & 32.37 & 32.29 & 32.37 \\
LNBL & 0.05 & 32.37 & 32.36 & 32.79 \\
(Low-Noise) & 0.10 & 32.37 & 32.37 & 61.53 \\
& 0.50 & 32.37 & 32.21 & 60.13 \\
& 1.00 & 32.37 & 31.60 & 75.70 \\
\hline & 0.01 & 11.54 & 11.32 & 11.51 \\
RNBL & 0.05 & 11.54 & 11.32 & 11.54 \\
(Real-Noise) & 0.10 & 11.54 & 11.55 & 15.94 \\
& 0.50 & 11.54 & 11.55 & 15.12 \\
& 1.00 & 11.54 & 3.34 & 4.67 \\
\hline
\end{tabular}

although it is not so pronounced. The best gain factors are usually achieved with the DWT-Wiener method.

Finally, for each method there exists an optimal sampling where the gain factor is maximum. For realistic sampling and SNR conditions (blue and orange lines in Fig. 5, this sampling is about $F W H M \sim 9$ pixels. By realistic conditions, we mean conditions similar to those in the GREAT08 challenge, i.e., data quality mimicking that of the EUCLID satellite in terms of sampling and $S N R$.

\subsection{Denoising of the GREAT08 challenge dataset}

The goal of the previous section was to test the effect of both denoising and sampling on the quality of the shape measurement of galaxies, in the absence of other numerical or instrumental disturbances. For this reason, the convolution of the galaxy images by the instrumental PSF is not included.

We now test the two most effective denoising methods, i.e., the DWT-Bayes and the DWT-Wiener, against the additional effect of PSF deconvolution and centroid shifts. This requires the use of a shape measurement method. For the sake of simplicity, we choose the KSB algorithm (Kaiser 1995) with the code developed by Catherine Heymans and Ludovic Van Waerbeke (Heymans et al. 2006), which is widely used, public, and efficient in terms of computing time. In addition, it does not rely on any fit of an arbitrary galaxy profile to the data.

The GREAT08 challenge dataset is ideal for carrying out our test in both low noise and real noise conditions. For this purpose, we use the whole low noise-blind (LNBL) dataset and a subset of 100 frames of the real noise-blind (RNBL) dataset (see the GREAT08 handbook for more detail, Bridle et al. 2009). The total number of galaxies used is therefore 15000 in low noise and 100000 in real noise conditions.

The PSF convolution, which modifies the effect of the noise on the galaxy shape measurement, makes it necessary to control the effect of denoising, prior to the shape measurements itself. We therefore introduce a "denoising strength" that allows us to fine-tune the amount of denoising of the data. In practice, we replace the noise mean standard deviation, $\sigma_{n}$, in Eqs. (1), (4), and (5), with a fractional mean standard deviation $\alpha \sigma_{n}$, where $0<\alpha \leq 1.0$.

We run the KSB method on the denoised GREAT08 data using three denoising strengths $\alpha=\{0.01,0.05,0.1,0.5,1.0\}$. The results are summarized in Table. 2 , where the quality factor, $Q$, is defined as in Bridle et al. (2010). High values of $Q$ indicate good shape measurements. As a sanity check, we run the KSB algorithm on the original noisy data as well and check that we obtain the same quality factors as in Bridle et al. (2010), i.e., $Q=32.37$ in low noise and $Q=11.54$ in real noise conditions.

The main result is that the DWT-Bayes method does not improve the quality factor and even degrades the shape measurement if full denoising $(\alpha=1.0)$ is used. The DWTWiener method improves the $Q$ factor by a factor of almost two in low noise conditions and by $35 \%$ in real noise conditions. Interestingly, we note that full denoising may degrade the results. One may instead apply partial denoising $(\alpha=0.1)$, which allows us to achieve a significant gain in quality factor without any significant risk of corrupting the data.

\section{Conclusions}

We have tested four different image denoising techniques on synthetic data of faint and small galaxies and we evaluate their effect on the shape measurement of galaxies in view of weak lensing studies. We have compared the performance of the algorithms for a range of $S N R$ and sampling conditions.

We found that simple median and Wiener filtering degrades the quality of the galaxy shape measurement unless very fine sampling is used. Local denoising methods such as wavelet filtering (DWT-Bayes) preserve the shape of galaxies in fine sampling condition but not for coarser sampling. However, a simple modification of the thresholding scheme of the wavelet method allows us to improve the $S N R$ of the data and the quality of the shape measurement. This new method, DWT-Wiener, consists of applying Wiener filtering to the wavelet coefficients rather than to the data themselves.

The DWT-Wiener method is tested on the GREAT08 challenge data in low noise and real noise conditions, showing an improvement of up to a factor of two (on the quality factor $Q$ ) over the shape measurement using the original, noisy, data.

Finally, we have shown that for a fixed $S N R$ there exist an optimal sampling of the galaxy images. For the typical $S N R$ expected in weak lensing space surveys, this sampling is about FWHM $\sim 9$ pixels. Satellites such as EUCLID or WFIRST will have a typical pixel scale of $0.1^{\prime \prime}$, allowing us to observe typical $z=1-2$ galaxies with almost this optimal sampling. This lends considerable hope to significantly improving future weak lensing measurements with image denoising.

Acknowledgements. The authors would like to thank C. Heyman and L. van Waerbeke for their KSB method code. The authors are also grateful to Malte Tewes for fruitful discussion. This work is supported by the Swiss National Science Foundation (SNSF).

\section{References}

Arce, G. R. 2005, Nonlinear Signal Processing: A Statistical Approach (New Jersey USA: Wiley)

Arias-Castro, E., \& Donoho, D. L. 2009, Ann. Statist., 37, 1172

Bacon, D. J., Réfrégier, A. R., \& Ellis, R. S. 2000, MNRAS, 318, 625

Bartelmann, M., \& Schneider, P. M. 2001, Phys. Rep., 340, 291

Bridle, S., Shawe-Taylor, J., \& Amara, A. 2009, Ann. App. Statist., 3, 6

Bridle, S., Balan, S. T., Bethge, M., et al. 2010, MNRAS, 405, 2044

Bruce, A., Donoho, D., \& Gao, H. 1996, Wavelet Analysis, IEEE Spectrum, 33, 26

Chang, S. G., Yu, B., \& Vetterli, M. 2000, IEEE Trans. Image Procss., 9, 1532

Daubechies, I. 1992, Ten Lectures on Wavelets, Society for Industrial and Applied Mathematics

Donoho, D. L., \& Johnstone, I. M. 1994, Biometrika, 81, 425 
Donoho, D. L., \& Johnstone, I. M. 1995, J. Stat. Assoc., 90, 1200 Frieman, J. A., Turner, M. S., \& Huterer, D. 2008, ARA\&A, 46, 385 Gonzalez, R. C., \& Woods R. E. 1992, Digital Image Processing (AddisonWesley)

Haar Zur, A. 1910, Math. Ann., 69, 331

Heymans, C., Van Waerbeke, L., Bacon, D., et al. 2006, MNRAS, 368, 1323

Howell Steve, B. 2006, Handbook of CCD Astronomy (Cambridge University Press)

Kaiser, N., Squires, G., \& Broadhurst, T. 1995, ApJ, 449, 460

Khireddine, A., Benmahammed, K., \& Puech, W. 2007, Adv. Eng. Software, 38, 513

Mallat, S. 1989, IEEE Trans. Pattern Anal. Mach. Intell., 11, 674

Massey, R., Rhodes, J., Ellis, R., et al. 2007a, Nature, 445, 286

Massey, R., Heymans, C., Bergé, J., et al. 2007b, MNRAS, 376, 13

Meyer, Y. 1990, Ondelettes et operateurs, I. Ondelettes (Paris: Hermann)
Perlmutter, S., Aldering, G., Goldhaber, G., et al. 1999, ApJ, 517, 565

Press, W. H., Flannery, B. P., Teukolsky, S. A., \& Vetterling, W. T. 2007, Numerical Recipes, The Art of Scientific Computing, 3rd edn. (Cambridge University Press)

Réfrégier, A., Amara, A., Kitching, T. D., et al. 2010 [arXiv: 1001.0061]

Riess, A. G., Filippenko, A. V., Challis, P., et al. 1998, AJ, 116, 1009

SciPy community 2010, SciPy Reference Guide Release, Release 0.9.0.dev6641 docs.scipy.org/doc/scipy/scipy-ref.pdf

Vetterli, M., \& Kovacevic, J. 1995, Wavelets and Subband Coding, Englewood Cliffs (NJ: Prentice Hall)

Van Waerbeke, L., Mellier, Y., Erben, T., et al. 2000, A\&A, 358, 30

Wiener, N. 1949, Extrapolation, Interpolation, and Smoothing of Stationary Time Series (New York: Wiley)

Wittman, D. M., Tyson, J. A., Kirkman, D., Dell'Antonio, I., \& Bernstein, G. 2000, Nature, 405, 143 